\title{
Beta blockers in heart failure with preserved ejection frac- tion and reactive airway disease
}

\begin{abstract}
Michael J. Scalese* Internal Medicine, University of South Alabama College of Medicine, USA

\section{Introduction}

In the United States alone, Heart Failure with Preserved Ejection Fraction (HFpEF) accounts for nearly five-hundred thousand admissions annually [1]. Despite medical therapy, patients with HFpEF have an estimated survival at one, three, and five years of $82 \%, 48 \%$, and $33 \%$ respectively [2]. As such, reducing morbidity and mortality in these patients has become increasingly important [3,4]. Guideline recommendations suggest pharmacological strategies used in heart failure with a reduced ejection fraction (HFrEF) may benefit those with a preserved ejection fraction [4]. However, most recommendations rely heavily on expert opinion as limited data exists to direct management of HFpEF [4].
\end{abstract}

Assistant Clinical Professor, Department of Pharmacy Practice, Auburn University Harrison School of Pharmacy and Adjunct Assistant Professor, Department of

Beta blockers are a main stay in the treatment of HFrEF due to an abundance of evidence supporting reduced hospitalizations and mortality [5-7]. However, use of beta blockers in patients with HFpEF have not been proven in clinical evaluations. Rather, large trials supporting beta blocker usage have major limitations restricting their application to practice $[8,9]$. Less robust data from observational and retrospective studies have demonstrated conflicting results further clouding the role of beta blockers in the preserved ejection fraction population [10-12]. Despite a lack of strong clinical data, guideline recommendations support beta blocker use in patients with HFpEF and no contraindications for use [4].

The clinical decision to use beta blockers must weigh the benefits against the risks of adverse effects such as hypotension, atrioventricular block, and respiratory complications. In patients with no underlying respiratory disease, beta blockers rarely exhibit clinical relevant respiratory effects [13]. However, in patient with pulmonary conditions the risks of beta-induced respiratory dysfunction is higher due to abnormal pathology and predisposition for complications [14]. Therefore, in patients with respiratory disease the risks for respiratory decompensation must be weighed against the potential benefit in HFpEF. The following clinical case and review of the literature describes a patient with concomitant $\mathrm{HFpEF}$ and reactive airway disease.

\section{Case}

A 35 year old African American male presented with hypercapnic hypoxemic respiratory failure and bilateral lower extremity edema resulting in admission to internal medicine services. The patient's past medical history was significant for HEpEF, hypertension, hyperlipidemia, type 2 diabetes mellitus and reactive airway disease. Approximately 3 months prior to current hospital admission the patient had an echocardiogram showing an ejection fraction of $55 \%$ and grade I diastolic dysfunction. Home medications included aspirin $5 \mathrm{mg}$ daily, furosemide $40 \mathrm{mg}$ daily, and pravastatin $20 \mathrm{mg}$ daily. All home medications were continued as an inpatient except atenolol (changed to metoprolol succinate $25 \mathrm{mg}$ daily) and furosemide (dose increased to $60 \mathrm{mg}$ IV twice daily).

Physical exam at presentation revealed expiratory wheezes and 4+ pitting edema bilaterally in the lower extremities but was otherwise within normal limits. On admission, the patient's vital signs were as follows: blood pressure $163 / 70 \mathrm{mmHg}$, heart rate 84 beats per minute, and respiratory rate 23 breaths per minute. His serum chemistry revealed: sodium $142 \mathrm{mEq} / \mathrm{L}$, potassium $3.9 \mathrm{mEq} / \mathrm{L}$, chloride 101 $\mathrm{mEq} / \mathrm{L}, \mathrm{CO}_{2} 42 \mathrm{mEq} / \mathrm{L}, \mathrm{BUN} 11 \mathrm{mg} / \mathrm{dL}$, Serum creatinine $1.66 \mathrm{mg} / \mathrm{dL}$, glucose $144 \mathrm{mg} / \mathrm{dL}$, calcium $7.7 \mathrm{mg} / \mathrm{dL}$, magnesium $1.7 \mathrm{mEq} / \mathrm{L}$. The initial arterial blood gas (ABG) showed: $\mathrm{pH} 7.40, \mathrm{pCO}_{2} 71 \mathrm{mmHg}$, $\mathrm{pO}_{2} 42 \mathrm{mmHg}, \mathrm{HCO}_{3} 43 \mathrm{mEq} / \mathrm{L}$. A chest $\mathrm{x}$-ray in the emergency department showed cardiomegaly and early changes indicative of pulmonary edema.

During the patient's hospital course, amlodipine was discontinued due to its potential role in the peripheral edema and the fosinopril was up-titrated for blood pressure control. Intravenous furosemide $60 \mathrm{mg}$ was used twice daily for 2 days with an appropriate diuretic response. Upon improvement in peripheral edema to $1+$ bilaterally (patient's baseline) furosemide was changed to $40 \mathrm{mg}$ by mouth daily. A repeat echocardiogram showed no changes from the previous study completed three months prior except for the presence of a trivial pericardial perfusion.

Pulmonary services were consulted and recommended discontinuing metoprolol due to worsening respiratory symptoms and scheduling nebulizer treatments with albuterol and ipratropium every 4 hours. The patient's symptoms and ABG showed marked improvement over the next 24 hours and the patient was restarted on metoprolol for his heart failure. His breathing continued to improve over the next Professor, Department of Pharmacy Practice, Auburn University Harrison School of Pharmacy and Adjunct Assistant Professor, Department of Internal Medicine, University of South Alabama College of Medicine, 650 Clinic Drive, Rm 2100 Mobile, AL 36688, USA, Tel: 251-445-9318; Fax: 251-445-9341; E-mail: mjs0065@auburn.edu

Key words: beta blockers, diastolic dysfunction, case report, heart failure with preserved ejection fraction ( $H F p E F)$, reactive airway disease

Received: February 07, 2015; Accepted: February 17, 2015; Published: February 20, 2015
$81 \mathrm{mg}$ daily, atenolol $25 \mathrm{mg}$ daily, fosinopril $10 \mathrm{mg}$ daily, amlodipine

Correspondence to: Michael J. Scalese, Pharm.D., BCPS, Assistant Clinical 
2 days resulting in discharge on nebulized albuterol solution with scheduled follow up with pulmonary and cardiology services.

\section{Discussion}

Although the patient case described is not an incredibly rare incident, it does describe a clinical dilemma for the management of patients with HFpEF and reactive airway disease. The risks of respiratory decompensation must be weighed against the perceived benefit of beta blockade in this subset of heart failure patients. Additional considerations such as beta blocker selectivity, choice of respiratory therapy, and severity of cardiopulmonary disease can help guide clinical decision making.

The use of beta blockers are endorsed by current guidelines for use in HFpEF patients despite limited data from randomized, controlled trials [4]. The recommendations are however, based highly on expert opinion and have a low level of evidence. It must be also be noted that while recommendations support use of beta blockers, its role is as an antihypertensive agent as opposed to their potential role in reducing morbidity and mortality [15]. In patients with a preserved ejection fraction and hypertrophic cardiomyopathy, a subset of HFpEF, guideline recommendations more strongly support beta blocker use [15]. However, the evidence for use in hypertrophic cardiomyopathy focuses on symptomatic improvement, especially with obstructive pathophysiology, rather than a mortality benefit [15].

No large, randomized, controlled, clinical trials have set out to evaluate the benefit of beta blockers in HFpEF. However, a prespecified subgroup analysis of the SENIORS trial assessed the benefit of nebivolol in HFpEF [8]. No difference was observed between nebivolol and placebo for the primary outcome of all-cause mortality or hospitalization for a cardiovascular reason (HR 0.81, 95\%CI:0.631.04) [8]. While no benefit was seen, the SENIORS study is the largest trial that has evaluated beta blockers in HFpEF. The limited difference in adverse effects and non-statistically significant reduction in cardiovascular outcomes compared with placebo suggest beta blockade may be a reasonable therapeutic option in heart failure patients with a preserved ejection fraction [8].

In the absence of other large clinical trials evaluating beta blocker therapy in HFpEF, observational data has been analyzed from a large Medicare database of patients hospitalized for heart failure [9]. Out of 24,689 total heart failure patients, 4,153 patients had a preserved ejection fraction. Beta blocker therapy at discharge was associated with improved survival at 1 year (HR 0.65, 95\%CI:0.57-0.73). However, after adjustment for baseline characteristics there was no significant difference in mortality or rehospitalization for heart failure. Analyses from smaller cohort data have failed to confirm these findings. Rather, studies described by Nevzorov et al. and El-Refai et al. suggest there may be reduced hospitalizations and all-cause mortality associated with beta blocker use irrespective of ejection fraction $[11,12]$. Nevzorov et al. also concluded that there was an association of reduced death $(p=0.001)$, hospitalization $(p=0.016)$, or a combination of both $(\mathrm{p}=0.009)$, in patients with HFpEF. Interestingly, a significant association was found in patients receiving traditional beta blockers for heart failure (metoprolol, carvediolol, bisoprolol) $(\mathrm{p}=0.014)$ as well as those receiving therapy with any beta blocker $(\mathrm{p}=0.009)$. This beneficial association from the smaller cohort evaluations favor the use of beta blockers in HFpEF. However, these finding were not consistent in all studies and the possible benefits must be weighed against the potential risks of using beta blockers.
An important risk to consider is the potential for respiratory compromise with blocking beta receptors in the pulmonary system. Beta blockers are generally considered to have minimal respiratory side effects in patients with no underlying pulmonary disease [13]. However, in patients such as the one described in the patient case, reactive airway disease may predispose patients to respiratory complications [14]. Cardiovascular selectivity of the beta-blockers used in this population is an incredible important consideration. Indeed, the distribution of beta receptors fluctuate by location in the pulmonary tree. The alveoli, containing $90 \%$ of the pulmonary beta receptors, are primarily beta2 whereas the larger airways are mostly beta-1 [14]. As such, beta-1 selective medications such as metoprolol and bisoprolol have lower risk of respiratory effects [14].

A recent meta-analysis including 32 randomized trials evaluated the risk for respiratory exacerbation with beta blocker use in reactive airway disease [14]. A profound reduction in respiratory function was observed with nonselective beta blockers [14]. Beta-1 selective drugs were also found to have a statistically significant risk of respiratory effects, though it was much less clinically relevant than the risk with nonselective beta blockers [14]. In addition, the presence of beta blockers attenuated patient's response to beta-2 bronchodilators with a two-fold higher effect with nonselective beta blockers than with beta1 selective ones [14]. The risks described in the meta-analysis suggest that beta 1-selective agents should be used with caution in patients with reactive airway disease.

\section{Conclusion}

Evaluations of beta-blockers in HFpEF have produced conflicting results making the role in therapy unclear. Nevertheless, guidelines endorse the use of beta blockers due to potential benefit especially in patients with low risk of adverse effects. Respiratory depression, a concerning adverse effect, is not commonly experienced unless the patient has underlying reactive airway disease. The respiratory risks in patients with reactive airway disease are more profound with nonselective beta blockers but may still be clinically relevant with beta1 selective drugs. Practitioners should weight the risks and benefits of beta blocker use to make individualized clinical decisions for patients with HFpEF and reactive airway disease.

\section{References}

1. Yancy CW, Lopatin M, Stevenson LW, De Marco T, Fonarow GC; ADHERE Scientific Advisory Committee and Investigators (2006) Clinical presentation, management, and in-hospital outcomes of patients admitted with acute decompensated heart failure with preserved systolic function: a report from the Acute Decompensated Heart Failure National Registry (ADHERE) Database. J Am Coll Cardiol 47: 76-84. [Crossref]

2. Gorelik O, Almoznino-Sarafian D, Shteinshnaider M, Alon I, Tzur I, et al. (2009) Clinical variables affecting survival in patients with decompensated diastolic versus systolic heart failure. Clin Res Cardiol 98: 224-232. [Crossref]

3. Banerjee P, Banerjee T, Khand A, Clark AL, Cleland JG (2002) Diastolic heart failure: neglected or misdiagnosed? J Am Coll Cardiol 39: 138-141. [Crossref]

4. Yancy CW, Jessup M, Bozkurt B (2013) ACCF/AHA guideline for the management of heart failure: a report of the American College of Cardiology Foundation/American Heart Association Task Force on Practice Guidelines. J Am Coll Cardiol 62: e147-239. [Crossref]

5. (1999) Effect of metoprolol $\mathrm{CR} / \mathrm{XL}$ in chronic heart failure: Metoprolol $\mathrm{CR} / \mathrm{XL}$ Randomised Intervention Trial in Congestive Heart Failure (MERIT-HF). Lancet 353: 2001-2007. [Crossref]

6. (1999) The Cardiac Insufficiency Bisoprolol Study II (CIBIS-II): a randomised trial Lancet 353: 9-13. [Crossref]

7. Packer M, Coats AJ, Fowler MB, Katus HA, Krum H, et al. (2001) Effect of carvedilo on survival in severe chronic heart failure. N Engl J Med 344: 1651-1658. [Crossref] 
8. Van Veldhuisen DJ, Cohen-Solal A, Bohm M (2009) Beta-blockade with nebivolol in elderly heart failure patients with impaired and preserved left ventricular ejection fraction: data from SENIORS (Study of Effects of Nebivolol Intervention on Outcomes and Rehospitalization in Seniors with Heart Failure). J Am Coll Cardiol 53: 2150-2158. [Crossref]

9. Hernandez AF, Hammill BG, O'Connor CM (2009) Clinical effectiveness of $\beta$-blockers in heart failure: findings from the OPTIMIZE-HF (Organized Program to Initiate Lifesaving Treatment in Hospitalized Patients with Heart Failure) registry. $J$ Am Coll Cardiol 53: 184-192. [Crossref]

10. Farasat SM, Bolger DT, Shetty V, Menachery EP, Gerstenblith G, et al. (2010) Effect of -blocker therapy on rehospitalization rates in women versus men with heart failure and preserved ejection fraction. Am J Cardiol 105: 229-234. [Crossref]

11. El-Refai M, Peterson EL, Wells K (2013) Comparison of $\beta$-blocker effectiveness in heart failure patients with preserved ejection fraction versus those with reduced ejection fraction. J Card Fail 19: 73-79. [Crossref]
12. Nevzorov R, Porath A, Henkin Y, Kobal SL, Jotkowitz A, et al. (2012) Effect of beta blocker therapy on survival of patients with heart failure and preserved systolic function following hospitalization with acute decompensated heart failure. Eur J Intern Med 23: 374-378. [Crossref]

13. Agostoni P, Palermo P, Contini M (2009) Respiratory effects of beta-blocker therapy in heart failure. Cardiovasc Drugs Ther 23: 377-384. [Crossref]

14. Gersh BJ, Maron BJ, Bonow RO (2011) ACCF/AHA guideline for the diagnosis and treatment of hypertrophic cardiomyopathy: a report of the American College of Cardiology Foundation/American Heart Association Task Force on Practice Guidelines. J Thorac Cardiovasc Surg 142: e153-203.

15. Morales DR, Jackson C, Lipworth BJ, Donnan PT, Guthrie B (2014) Adverse respiratory effect of acute $\beta$-blocker exposure in asthma: a systematic review and metaanalysis of randomized controlled trials. Chest 145: 779-786. [Crossref]

Copyright: $\mathbb{C} 2015$ Scalese MJ. This is an open-access article distributed under the terms of the Creative Commons Attribution License, which permits unrestricted use, distribution, and reproduction in any medium, provided the original author and source are credited. 